\title{
Análisis de la situación de la pesca artesanal en el delta y valle bajo del río Senegal a partir del marco conceptual DPSIR ${ }^{1}$
}

\section{Analysis of the situation of artisanal fisheries in the delta and lower valley of the Senegal River from the DPSIR conceptual framework}

\author{
Elhadji Oumar Touré ${ }^{2}$, Francesc Romagosa Casals ${ }^{3}$ \\ y Françoise Breton Renard ${ }^{3}$
}

\section{INTRODUCCIÓN}

Durante las últimas décadas se han realizado distintos estudios en el ámbito de la gestión de la pesca, tanto en el ámbito marino como en el terrestre. El concepto de gestión racional de los recursos pesqueros se ha utilizado en muchos países con el fin de luchar contra el colapso de las reservas. Los modelos biológicos (Schaefer, 1954) y bioeconómicos (Gordon, 1954; Hardin, 1968; UICN, 2006) se centran en estudiar la sostenibilidad de la actividad según la relación existente entre los artes de pesca y las capturas, dejando en un

\footnotetext{
${ }^{1}$ Este estudio fue posible gracias a la financiación del Laboratorio Mixto Internacional Patrimonio y Territorio del Agua (LMI PATEO) del Instituto de Investigación para el Desarrollo (IRD) de Francia en Senegal. También queremos agradecer al Grupo de Investigación en Recursos Costeros y Paisaje (INTERFASE) del Departamento de Geografía de la Universidad Autónoma de Barcelona, a través del cual se financió el trabajo de campo.

2 Instituto de Ciencia y Tecnología Ambientales, Universidad Autónoma de Barcelona. elhadjioumar.toure@e-campus@uab.cat

${ }^{3}$ Departamento de Geografía, Universidad Autónoma de Barcelona. francesc.romagosa@uab.cat
} 
segundo plano el medio biótico y abiótico explotados por los pescadores (Rigler, 1982). Ante el colapso de las reservas pesqueras, con el fin de buscar el paradigma capaz de resolver el declive del sector, las disciplinas socio-antropológicas han analizado la actividad teniendo en cuenta el espacio haliéutico, el impacto del cambio climático, la mejora en la tecnología y la demanda del mercado, entre otros aspectos (Corlay, 1993; Le Fur et al., 1999; Mbaye, 2002; Touré y Breton, 2013).

Hoy en día, el nuevo contexto de la gestión de la pesca artesanal se caracteriza por una situación globalizada en la que la integración de los factores naturales y socioeconómicos es un factor cada vez más importante (Quensière, 1993). La dimensión compleja de las pesquerías determina que su análisis e interpretación no pueda abordarse estrictamente desde enfoques unidisciplinares y parcelarios. En este sentido, resulta necesario plantear enfoques holísticos como los que ofrecen la Geografía o las Ciencias Ambientales, para intentar superar, en la medida de lo posible, la fragmentación del conocimiento y los análisis sesgados.

El marco conceptual DPSIR (del inglés Drivers, Pressures, State, Impacts \& Responses, es decir, fuerzas inductoras, presiones, estado, impactos y respuestas), diseñado por la Agencia Europea del Medio Ambiente (EEA, 1999; Svarstag et al., 2008) proporciona un marco integrado de análisis muy adecuado para el problema que nos ocupa. El modelo DPSIR, de hecho, se utiliza para evaluar y gestionar los problemas ambientales y describir las relaciones entre orígenes y consecuencias de esos problemas con el fin de entender su dinámica. En muchos lugares en el mundo, se han experimentado y elaborado indicadores de sostenibilidad y de gestión de la pesca artesanal utilizando este modelo (Bidone et al., 2004; Camanho et al., 2010).

La pesca, desde el punto de vista de los ingresos de la mano de obra que emplea, es sin duda la actividad económica más importante de la cuenca del río Senegal, después de la agricultura, especialmente para las poblaciones que habitan cerca del río en el delta y el valle bajo, en Senegal (Magrin y Seck, 2009). Sin embargo, actualmente se cuestiona seriamente el futuro de este sector en toda la región, ya que desde hace unos años se va notando una disminución continua de las cantidades capturadas y el rendimiento por arte de pesca. Las capturas han ido bajando paulatinamente, pasando de las 35.000 toneladas en 1956 hasta las 8.000-10.000 actualmente (Bousso, 1997; Bâ, 2013) (figura 1). Paralelamente, la población ha ido aumentando a un ritmo elevado (estimada en 230.669 habitantes en 2010 en la provincia de SaintLouis), ocasionando problemas para satisfacer una demanda de pescado estimada en $35 \mathrm{~kg} /$ persona/año (SRSD, 2010). 
FIGURA 1

EVOLUCIÓN DE LAS CAPTURAS DE PESCADO EN EL DELTA Y EL VALLE BAJO DEL RÍO SENEGAL (1950-2010)

(T)

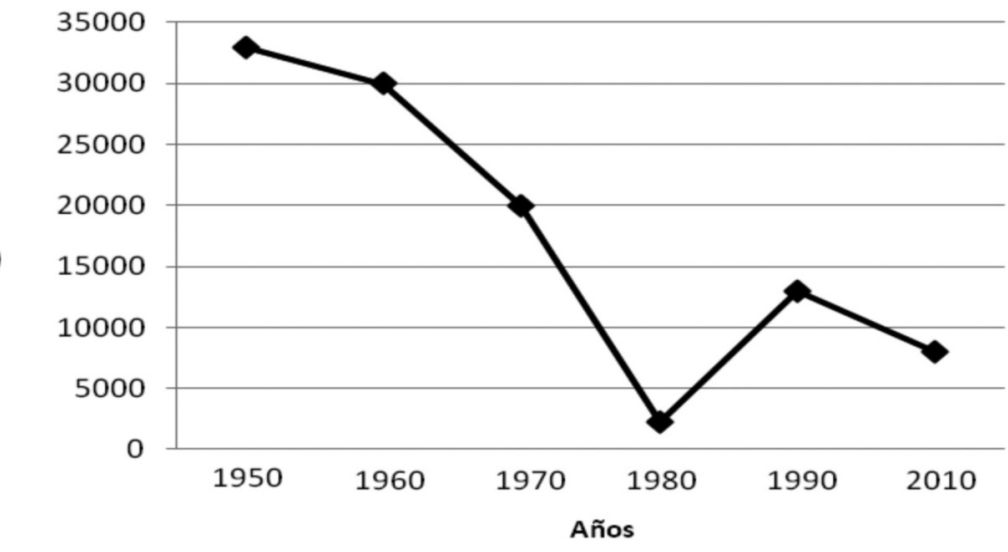

Fuente: Elaboración propia a partir de Bousso (1997) y Kane (2010).

Ciertos autores que han estudiado la región (por ejemplo, OMVS, 2005; Mietton et al., 2007; Camara, 2008; Kane, 2010) relacionan este fenómeno con los proyectos de desarrollo y transformación del río y su cuenca baja (presas, diques, canales de drenaje del agua, etc.) y a sus impactos medioambientales (disminución significativa de la salinidad al norte de la presa de Diama, proliferación de algas flotantes, eutrofización, contaminación, etc.). En este escenario, los cambios en los usos del suelo en el delta, determinados tanto por factores biofísicos (variabilidad del clima) como, de forma especialmente notable, por fuerzas inductoras de carácter socioeconómico, parecen tener mayor trascendencia causal en la modificación de las pesquerías artesanales. Al mismo tiempo, la biodiversidad y las capturas por arte de pesca se confirman como un indicador excepcional a la hora de poner de manifiesto sus impactos.

En el área de estudio, hay pocos estudios que aborden el análisis de la pesca artesanal de manera global integrando las dimensiones bioecológicas y socioeconómicas. Este artículo intenta paliar esta carencia. El trabajo tiene como objetivos específicos: (1) generar indicadores ambientales y socioeconómicos aplicables a la pesca fluvial de la zona; (2) desarrollar y relacionar los diferentes elementos del modelo DPSIR; (3) hacer un inventario del estado de las especies presentes y evaluar su abundancia relativa en relación con la calidad del agua; y (4) generar conocimiento para favorecer una gestión más sostenible del sector. 
Para lograr estos objetivos, la revisión de los elementos del modelo DPSIR en la literatura nos ha permitido destacar como fuerzas motrices o inductoras (D), los aspectos socio-económicos y demográficos (Mangi, 2007). En un estudio llevado a cabo por la Organización para la Cooperación y el Desarrollo Económico (OECD, 1994) se consideran como fuerzas inductoras la demanda del mercado en pescado, el valor mercantil, la mejora tecnológica y la necesidad de los recursos hídricos de conducción. Por contra, las presiones (P) son las acciones humanas que pueden causar cambios ambientales. Maxim et al. (2009) consideran las distintas acciones humanas como factores causantes de daños y/o degradación del entorno. Los indicadores de estado (S) muestran los cambios en los ecosistemas explotados, analizando las modificaciones químicas, físicas y biológicas relacionadas, teniendo en cuenta al mismo tiempo la dimensión social. Por su lado, los impactos (I) se definen como los efectos tanto positivos como negativos de las actividades humanas que se encuentran en el ámbito natural, social y económico (Elliot, 2002). Finalmente, se consideran como respuestas $(\mathrm{R})$ todas las medidas (preventivas, curativas y adaptativas) implementadas, directamente en las categorías D, P, S o I, por parte de la población o del estado con el fin de mejorar el sistema (Lin et al., 2007).

\section{ÁREA DE ESTUDIO}

El área de estudio corresponde a una región ecológica que se extiende desde la ciudad de Podor hasta la desembocadura del río Senegal, en la provincia de Saint-Louis (noroeste de Senegal). Desde el punto de vista del paisaje, en el área de estudio se pueden diferenciar, según las características hidrológicas, hidrodinámicas y sedimentarias, tres ecosistemas, que son: el valle bajo, que se extiende de Podor hasta Richard-Toll; el delta, que se encuentra entre Richard y la presa de Diama; y la zona de estuario que va desde la presa de Diama hasta la propia desembocadura del río (figura 2).

El área de estudio se desarrolló geológicamente en la parte de las extensas llanuras senegalo-mauritanias. Según Kane (1997), en la fase árida del oligoceno (20.000 antes de nuestra era), las dunas de arena adoptaron una forma en cadena con orientación NE-SO, y cerraron el acceso al mar, por lo que impusieron un régimen endorreico en el valle. A esta fase de terraplenado le sucede una fase pluvial que encauza el río a través de las dunas hasta llegar el mar (Sall, 1982). Debido a una abundante sedimentación posterior, el río construyó grandes taludes a su paso. En una fase aún posterior, el río serpenteaba y trazaba meandros a causa del hundimiento progresivo de los terraple- 
FIGURA 2

LOCALIZACIÓN DEL ÁREA DE ESTUDIO

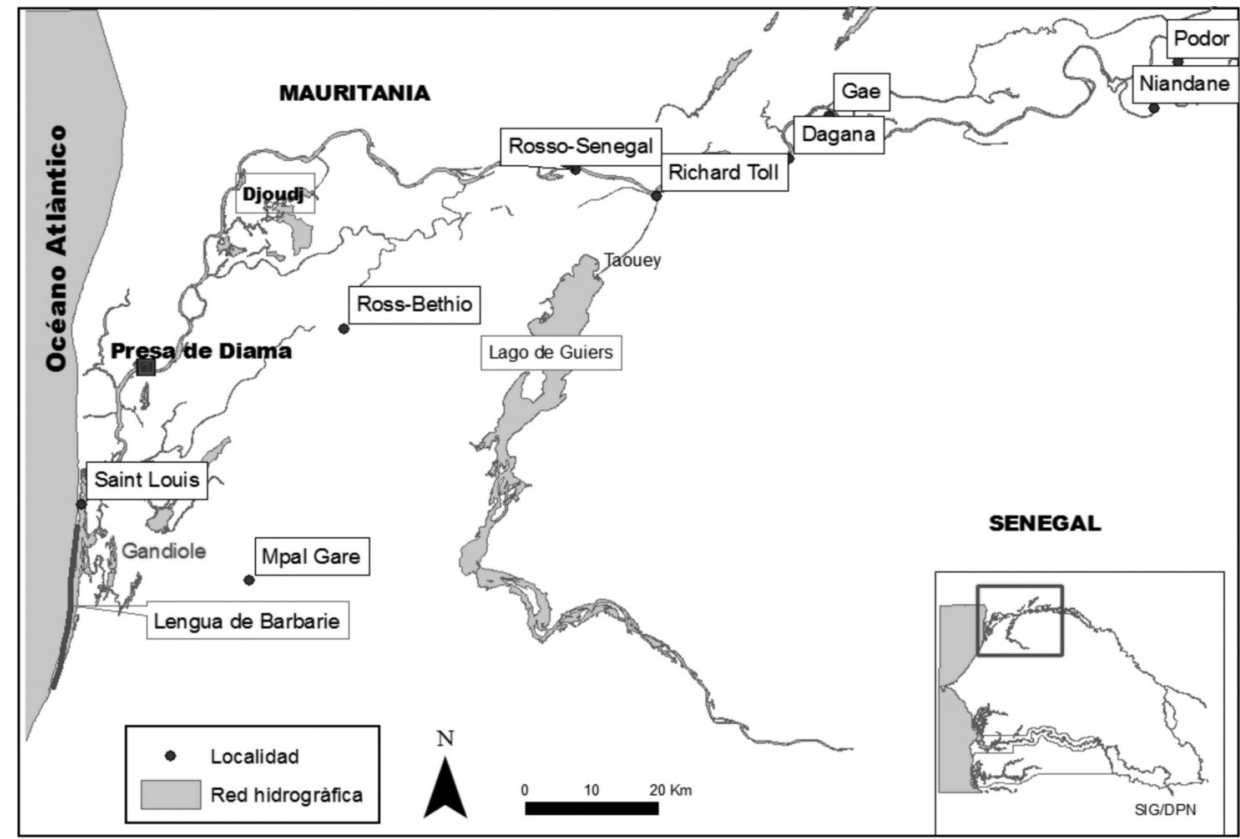

Fuente: Elaboración propia a partir de la base de datos de la dirección de los parques de Senegal.

nes, y, finalmente dio lugar a sistemas de pequeñas elevaciones y otros cúmulos deltaicos en los que las corrientes de agua depositan lentamente sus arcillas hasta formar las depresiones o sebjas (Faye, 1996). De forma particular, de un lado al otro de su trayecto, el río alimenta dos depresiones naturales (el lago Rkiz en Mauritania y el lago de Guiers en Senegal), así como una serie de remansos y estanques (Ndiael, Djoudj, Gorom-Lampsar, Djeuss) que se caracterizan por sus suelos salinos (Monteillet, 1988).

El régimen hidrológico, fuertemente modificado por la presa de Diama y el canal de la Lengua de Barbarie, han acelerado la salinización de las aguas subterráneas del valle bajo del río, generalmente utilizadas para el abastecimiento humano y la producción agrícola. Según Kane (2010), los hidrólogos coinciden en afirmar que para que exista una buena recarga de los acuíferos se precisa una precipitación total anual de $400 \mathrm{~mm}$. Sin embargo, el promedio en la estación de Saint-Louis por el periodo de 1968-2010 fue de 236,37 mm (Bâ, 
2013). Estos cambios socioambientales contribuyen enormemente a la vulnerabilidad de las comunidades locales.

La actividad económica local se basa principalmente en la agricultura, la pesca, la ganadería, el turismo, la explotación de sal y el comercio. Estas actividades contribuyen hasta el $62 \%$ del PIB del departamento de Saint-Louis (Bâ, 2013). Aparte de la pesca industrial en el Océano Atlántico, también hay una pesca tradicional practicada no sólo en el mar, sino también en el río Senegal, en el lago de Guiers y en diferentes arroyos y estanques por parte de los pescadores Guet ndariens, Cuballos, Lebous, entre otros. El turismo es un sector importante en la economía local, siendo los principales atractivos la ciudad de Saint-Louis (con un importante patrimonio histórico, la ciudad fue declarada Patrimonio de la Humanidad en 2000) y los cercanos parques nacionales de Djoudj, Geumbeul y la Lengua de Barbarie, ecosistemas de un elevado valor ecológico que forman parte de la reserva transfronteriza Djoudj-Diawling (Diarra, 2003; Touré y Romagosa, 2013). La actividad económica en el resto de la región está dominada por la agricultura y la ganadería. La agricultura se desarrolla sobre terrenos húmedos cercanos a los cursos fluviales, llamados Walo y Niayes o bien en la zona más interior y árida, en forma de agricultura de secano. Los principales cultivos alimentarios son el arroz, el mijo, el sorgo, el maíz, la papa y el frijol dulce, mientras que los cultivos industriales son la caña de azúcar, el tomate y la cebolla, cultivados en Richard-Toll y su entorno. La ganadería, con la cría de ovejas, cabras y vacas, y la comercialización de los productos ganaderos (carne, leche, piel), la ejerce mayoritariamente la etnia Fulbe, sobre todo en los municipios de Ross-Bethio, Rosso y Podor. La explotación y la comercialización de la sal son muy importantes en los municipios situados en la región de Gandiole y constituye una proporción de ingresos relativamente importantes para la población local.

\section{Metodología y FUENTES DE INFORMACiÓN}

La metodología utilizada para analizar la situación de la pesca artesanal se basa, por un lado, en adaptar los conceptos o indicadores del DPSIR al contexto local del área de estudio. No obstante, al mismo tiempo se destaca la necesidad de tratar de integrar y complementar los indicadores biofísicos y socioeconómicos dentro del diseño experimental para poner de manifiesto las interrelaciones entre los componentes del sistema. La principal dificultad estriba en que los socioecosistemas de la cuenca del río Senegal son sistemas complejos caracterizados por varias discontinuidades (zona costera, estuario, 
delta, etc.). Este hecho, comporta un esfuerzo metodológico para la elección de los indicadores más relevantes teniendo en cuenta que cada elemento del DPSIR puede a su vez convertirse en otra parte del sistema.

La caracterización del sistema pesquero se hizo a partir del trabajo de campo y de análisis de los registros de aprovechamiento contenidos en el centro de investigación oceanográfico de Dakar-Thiaroye, del Ministerio de Pesca y Economía Marítima de Senegal, la Dirección de Pesca continental y Acuicultura (DPCA) y el archivo del Instituto de la Pesca y de la Acuicultura (IUPA). En este sentido, el análisis de la situación se ha orientado a la caracterización de la estructura y la dinámica de la actividad para la obtención de datos a través de dos censos durante un ciclo anual de pesca: un censo en temporada seca (noviembre-julio) y otro en temporada de lluvia (agosto-octubre).

De este modo, el plan de encuesta se articuló en tres partes: $1^{a}$ ) Enfoque descriptivo sobre los artes de pesca y pueblos de pescadores con el fin de identificar grupos homogéneos en el territorio y tipos de presiones sobre los ecosistemas; $2^{a}$ ) Estudio de la dinámica de la actividad pesquera y su resultado, con las capturas hechas por los diferentes tipos de artes más representativos en el área; y $3^{a}$ ) Contabilidad de la biodiversidad de peces según una zonación ecológica que separa el norte del sur de la presa de Diama.

\section{FIGURA 3}

\section{MODELO INTEGRADO PARA EL ESTUDIO DE LA PESCA ARTESANAL, BASADO EN EL MARCO CONCEPTUAL DPSIR}

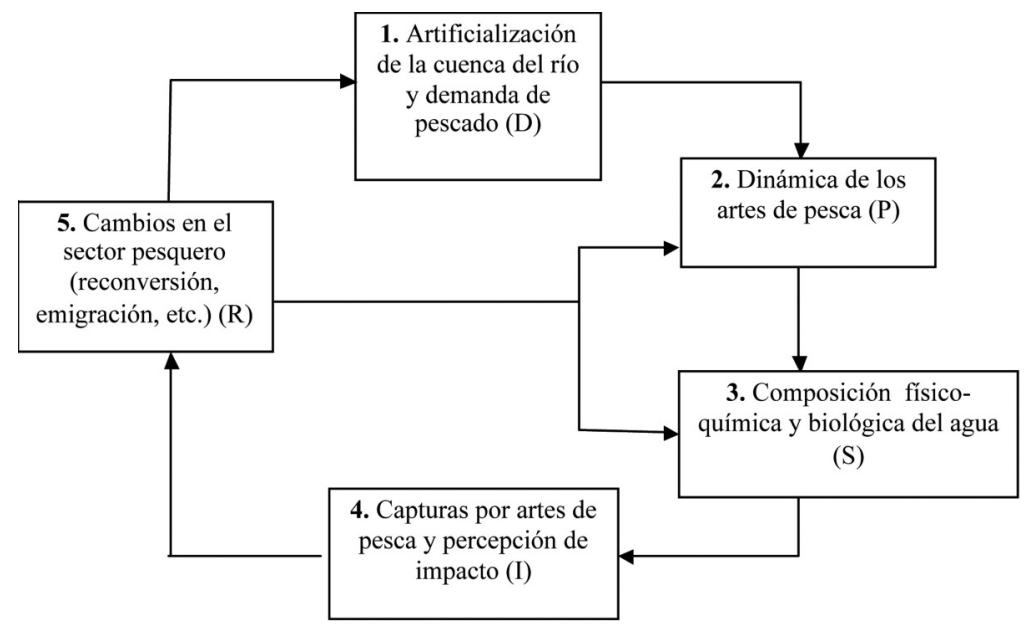

Fuente: Elaboración propia. 
La recolección de datos sobre el agua y su calidad físico-química se hizo a través de la base de datos del Laboratorio de Geomorfología e Hidrología de la Universidad de Dakar, la Sociedad Nacional de Gestión y Explotación de las Tierras del Delta (SAED), la Organización para el Desarrollo del río Senegal (OMVS) y algunos estudios realizados en la cuenca (Faye, 1996; Kane, 2010; Bâ, 2013). La parte sincrónica para implementar los indicadores se realizó aplicando el esquema DPSIR (figura 3).

La artificialización de la cuenca y la demanda de pescado son los indicadores calculados para las fuerzas inductoras (D). Un régimen artificial es un conjunto de instalaciones y operaciones a partir de las cuales el sistema hidrológico se transforma, domestica y el suministro de agua y el aprovechamiento humano depende exclusivamente de presas, canales, diques, remansos, etc. Como variables potencialmente de fuerzas inductoras socioeconómicas se realizó un inventario de infraestructuras, considerando el perímetro y/o la parcela hidroagrícolas (ha o $\mathrm{km}$ ) y la demanda de pescado, la evolución de los precios (FCFA/kg/especie).

La dinámica de los artes de pesca que explotan los ecosistemas es el principal elemento que ejerce presiones sobre el recurso (P). Los horarios de pesca (horarios de ida, vuelta y duración), el número medio de artes utilizados por cada pueblo, constituyen variables para medir la intensidad según el territorio de pesca y el pescado que se pretende capturar. Para el análisis se utilizó como herramienta el programa Spad, así como la modelización espacial con el objetivo de reducir el número de variables (16 artes), describir la estructura subyacente entre variables y clasificarlas en grupos (análisis factorial).

Los cambios en la composición físico-química y biológica del agua son, por su parte, los indicadores empleados para evaluar el estado del área explotada (S). Las variables fisicoquímicas como la salinidad (g/l), el nivel de $\mathrm{pH}$ y la abundancia biológica (con valores que van de 0 -especie extinta- a 5 -especie muy abundante-) permiten medir la evolución de la diversidad ecológica (Bousso y Touré, 2008).

Las capturas por arte de pesca constituyen los indicadores de impacto (I). La variable número de $\mathrm{kg}$ de pescado capturado por arte y operación de pesca nos permite conocer el impacto de la actividad. Para el seguimiento se utilizaron como muestreo los siete tipos de arte más representativos (aquellos que constituyen más del 90\% del potencial de pesca de la zona: goubole, saina, sabel, rauk, fele fele, halcón, palangra). Además de los datos sobre el impacto, se incorporaron fuentes orales sobre la percepción de los pescadores sobre los impactos de la presa de Diama y del canal de la Lengua de Barbarie. 
Finalmente, los indicadores de respuesta (R) escogidos fueron las estrategias desarrolladas por los pescadores con el fin de adaptarse a los cambios o para mejorar la actividad. El diagnóstico del calendario de actividad de los pescadores nos permite conocer la reconversión de los pescadores hacia otras actividades, las migraciones, etc. Las entrevistas se realizaron sobre una muestra de 200 pescadores (100 pescadores distribuidos entre las estaciones de pesca de Saint-Louis y Gandiole en la zona del estuario y 100 en las estaciones de Richard-Toll y Podor-Matam, ya en sector de agua dulce).

\section{RESUlTADOS}

\section{Artificialización de la cuenca y demanda de pescado (D)}

Los procesos biológicos (población de una especie, juveniles, crecimiento, abundancia, etc.) son especialmente sensibles a las variaciones del medio natural. Antes de la construcción de las grandes infraestructuras hidroagrícolas, durante la estación de lluvias, el río bajaba con mucha agua dulce que barría la sal hacia el mar. El régimen del río se caracterizaba por precipitaciones abundantes, afluentes importantes y la invasión marina era evidente hasta unos $200 \mathrm{~km}$ tierra adentro desde la desembocadura. El régimen tradicional del río era de tipo tropical húmedo, con una temporada de crecidas del nivel de las aguas (entre junio-julio y octubre-noviembre) con caudales que podían llegar hasta 5000 m.3s-1 en el máximo de la temporada y, una temporada de bajada del nivel de las aguas que podía durar hasta siete meses (de diciembre hasta junio). Este don del río permitía pescar sin problemas, así mismo aseguraba la recarga de los acuíferos, así como de los remansos, y lagos, que tenían agua dulce, incluso durante la estación seca (Kane, 2010; Touré y Romagosa, 2013). Durante este periodo, las capturas anuales de pesca eran relativamente importantes y siempre superiores a 20.000 toneladas, pudiendo llegar a las 35.000 toneladas (figura 1).

Los años de sequías que vinieron durante las siguientes décadas (19702000), así como los imperativos del desarrollo sostenible condujeron a una tendencia de intensificación de los usos del agua con la generalización de la agricultura con el fin de permitir la autosuficiencia alimentaria y aumentar los ingresos de la población. En este sentido, durante los últimos años se han materializado en el área del delta y valle bajo del Senegal distintas obras hidráulicas (remansos, canales, diques, presas) que suman 33.700 hectáreas de nuevos espacios irrigados, gestionados y planificados por la entidad pública SAED, anteriormente mencionada. 
A partir de la artificialización del régimen hidrológico en el área se pasó a depender en parte de las lluvias y en parte del modo de gestión de las infraestructuras. En efecto, la construcción de la presa de Diama en 1986, pensada para irrigar las zonas más interiores y posibilitar una agricultura intensiva dominada por el arroz, provocó una alteración en el ciclo hidrológico, bloqueando la subida del agua salada hacia el valle. Por consiguiente, se pasó de un medio acuático salino y salobre con marcados cambios estacionales, a una ecología perenne de agua dulce, de flujo bajo. La presa queda cerrada los 8 meses del año, en este sentido, el régimen estuárico de la parte baja del delta se transforma en un sistema hidrológico homogéneo, de tipo laguna.

Existe, desde luego, una relación causa-efecto entre fuerzas inductoras naturales y socioeconómicas y viceversa. Los cambios comentados han ocasionado la degradación de la población de peces de la que disponen los pescadores (cantidad y calidad). La disponibilidad, de agua dulce en el norte del delta, a partir de la construcción de la presa de Diama (en comparación con el sector estuárico situado al sur y de carácter salino), en cantidad suficiente (para la agricultura de regadío, el consumo doméstico, las agroindustrias, etc.) comportó un aumento de la población por inmigración y, por lo tanto, aumentó también la demanda de pescado. La pesca, como fuente de empleo e ingresos, tiene en los precios un indicador muy importante y constituye una de las fuerzas inductoras que determina y explica en parte la dinámica de la propia actividad, que puede generar presiones sobre los ecosistemas. Las especies que se pretenden capturar son un factor importante que generalmente viene determinado por los precios del mercado. En este sentido, el estudio realizado sobre la evolución de los precios del pescado durante el año 2013 permitió identificar los cambios en las especies más emblemáticas. En el periodo seco (de noviembre hasta julio), el pez bonga (Ethmalosa fimbriata) y la alacha (Sardinella aurita) dominan en las capturas en Saint-Louis y constituyen la principal fuente de ingresos por parte de los pescadores. Los precios medios, oscilan entre los 45 y 106 FCFA/kg y 68 y 150 respectivamente en el puerto pesquero de Saint-Louis. Por su parte, la pesca más preciada, el langostino blanco (Penaeus notialis), se captura en la desembocadura, al borde del canal de la Lengua de Barbarie, y más hacia el norte, en Mauritania. En los puertos pesqueros interiores de Richard-Toll y Podor, la perca del Nilo (Lates niloticus), el Ndiaguel (Heterotis niloticus) y la tilapia del Nilo (Oreochromis niloticus) son las especies preferidas por los pescadores y la población local, ya que los precios oscilan entre los 450 y los 600 FCFA/kg (cuadro 1). 


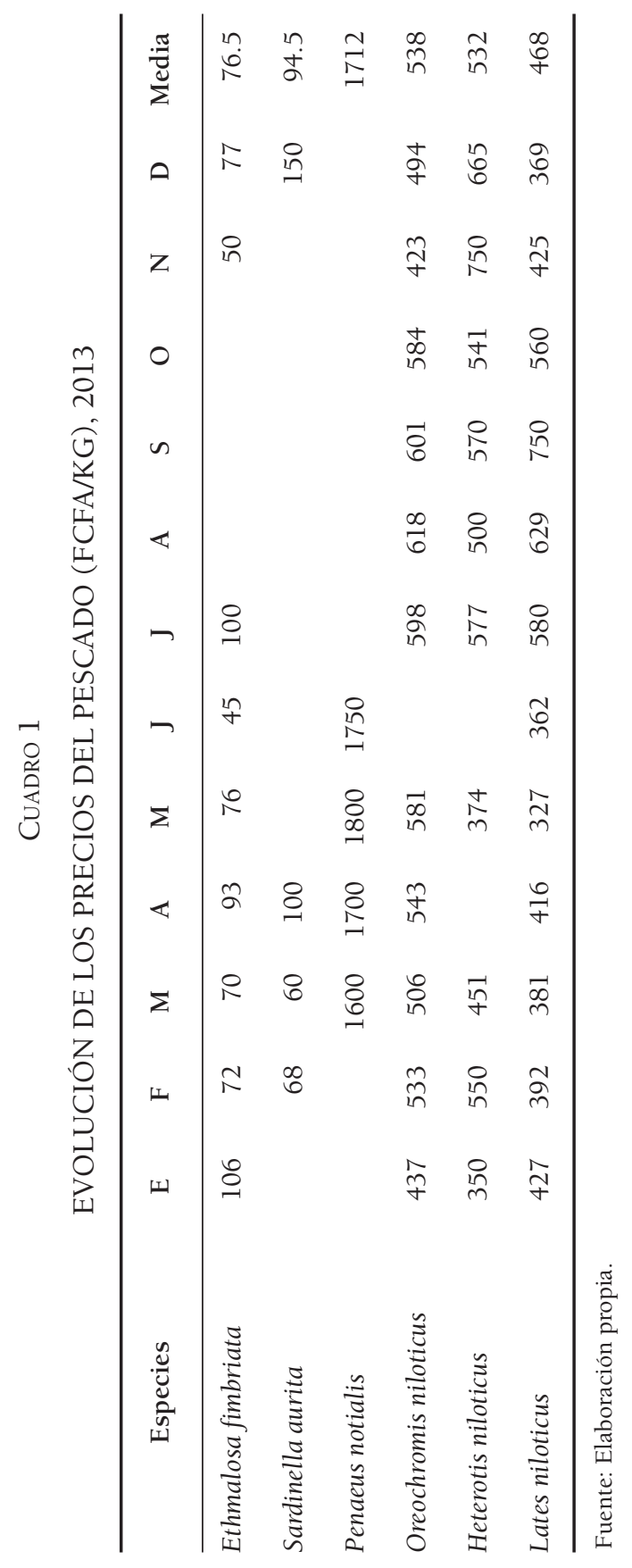

Estudios Geográficos, Vol. LXXVI, 279, pp. 703-731, julio-diciembre 2015 ISSN: 0014-1496, eISSN: 1988-8546, doi: 10.3989/estgeogr.201525 


\section{Presiones sobre los ecosistemas y los recursos pesqueros (P)}

Los pescadores utilizan distintos artes para pescar. Los artes de pesca son artesanos, elaborados con materiales locales y su uso depende de las especies que se pretenden capturar y de la época del año. Para analizar los tipos de presión sobre los ecosistemas, primero se hizo un censo de todos los artes que operan en las distintas localidades, luego una descripción de los artes de pesca y, en tercer lugar una tipología de los artes de pesca según la localidad, con el objetivo de detectar los tipos de concentración espacial y temporal. El censo efectuado en las poblaciones de pescadores permitió conocer con exactitud el número de artes de pesca y los pescadores que se dedican a otras actividades. En cualquier caso, cuando hablamos de pescadores nos referimos exclusivamente a los que tienen el material para pescar de manera permanente, ya sean pescadores profesionales o temporales. En nuestro recorrido por la zona de estudio contabilizamos un total de 1056 artes de pesca en enero y 982 en octubre. La figura 4 permite observar la distribución de estos artes de pesca por poblaciones.

FIGURA 4

DISTRIBUCIÓN TERRITORIAL DE LOS ARTES DE PESCA SEGÚN LOS PUEBLOS DE PESCADORES

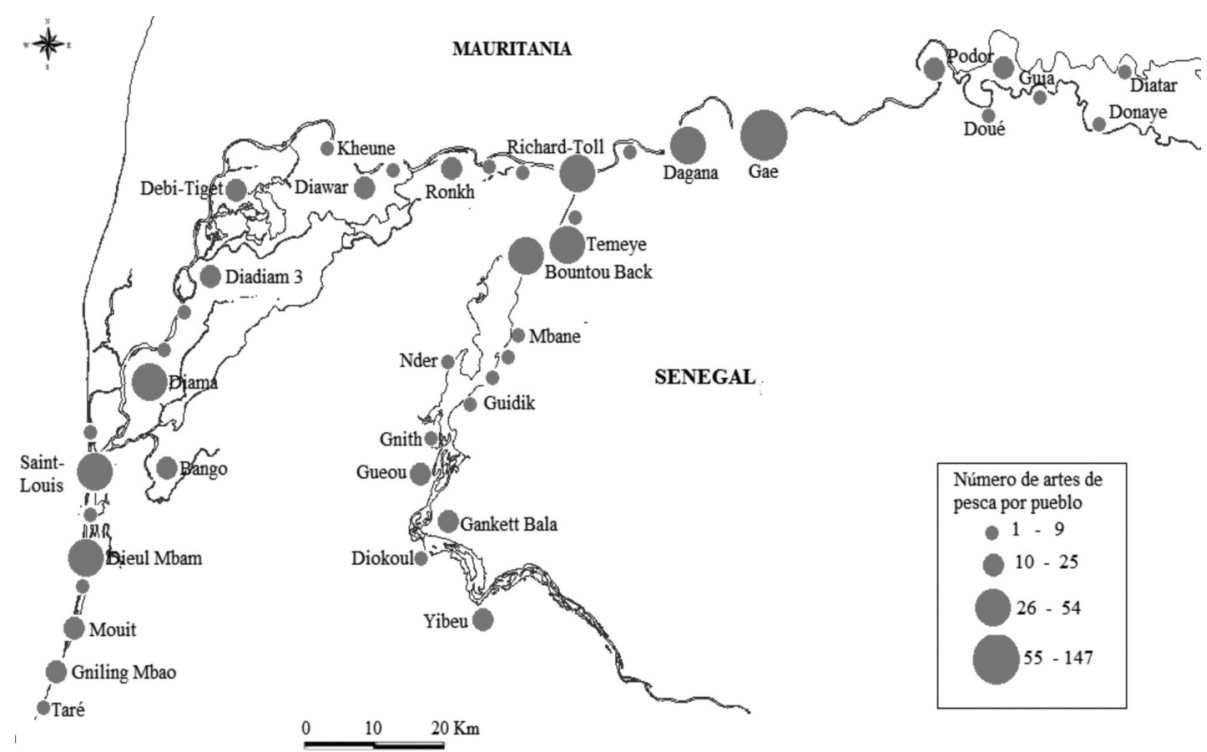

Fuente: Elaboración propia.

Estudios Geográficos, Vol. LXXVI, 279, pp. 703-731, julio-diciembre 2015 ISSN: 0014-1496, eISSN: 1988-8546, doi: 10.3989/estgeogr.201525 
Características de los artes de pesca que ejercen presión sobre los ecosistemas

Generalmente los pescadores disponen de varios artes y una embarcación con la que van a pescar, según las especies que pretenden capturar, el lugar de pesca y el periodo del año. Actualmente, los artes de pesca formados por redes son los mayoritarios. Unos operan en la superficie, algunos a media agua y otros en el fondo. Por lo general, encontramos 18 artes de pesca en red y 3 tipos distintos de embarcaciones asociadas a estos artes. Su análisis y descripción nos permitió comprender mejor las diferentes técnicas que usan los pescadores, para poder elaborar posteriormente una tipología.

Dentro del uso de las redes destaca el procedimiento denominado «pesca de arrastre en pareja», técnica que se realiza con dos barcos, uno a cada lado de la red, para mantenerla abierta. Cuando la red está próxima a la orilla, un pescador se mete al mar y junta las dos alas para formar el copo en la parte media de la red que, con sus plomos, ha venido arrastrando todo el fondo, capturando el pescado.

También se utilizan las redes fijas (aquellas que se colocan en un lugar determinado de la costa para interceptar el paso de los peces), así como las artes de deriva. En este último caso, se trata de artes de enmalle formadas por piezas rectangulares de red de nylon. Se calan en el río sin fondearlas, dejándolas a merced de las corrientes, sostenidas en posición vertical mediante flotadores.

Las redes de cerco, por su parte, se utilizan para la captura de peces cuya costumbre es nadar formando densos cardúmenes o bancos de peces, ya sea en la superficie o a media agua. En cuanto a los artes de arrastre, se utilizan para la pesca de los organismos que viven en el fondo. Actualmente, aparte de las redes mencionadas se siguen utilizando artes tradicionales, como los aparejos (artes de pesca formadas por un cordel con un anzuelo en su extremo o a veces sin él), el esparavel, las trampas y los arpones (cuadro 2). 


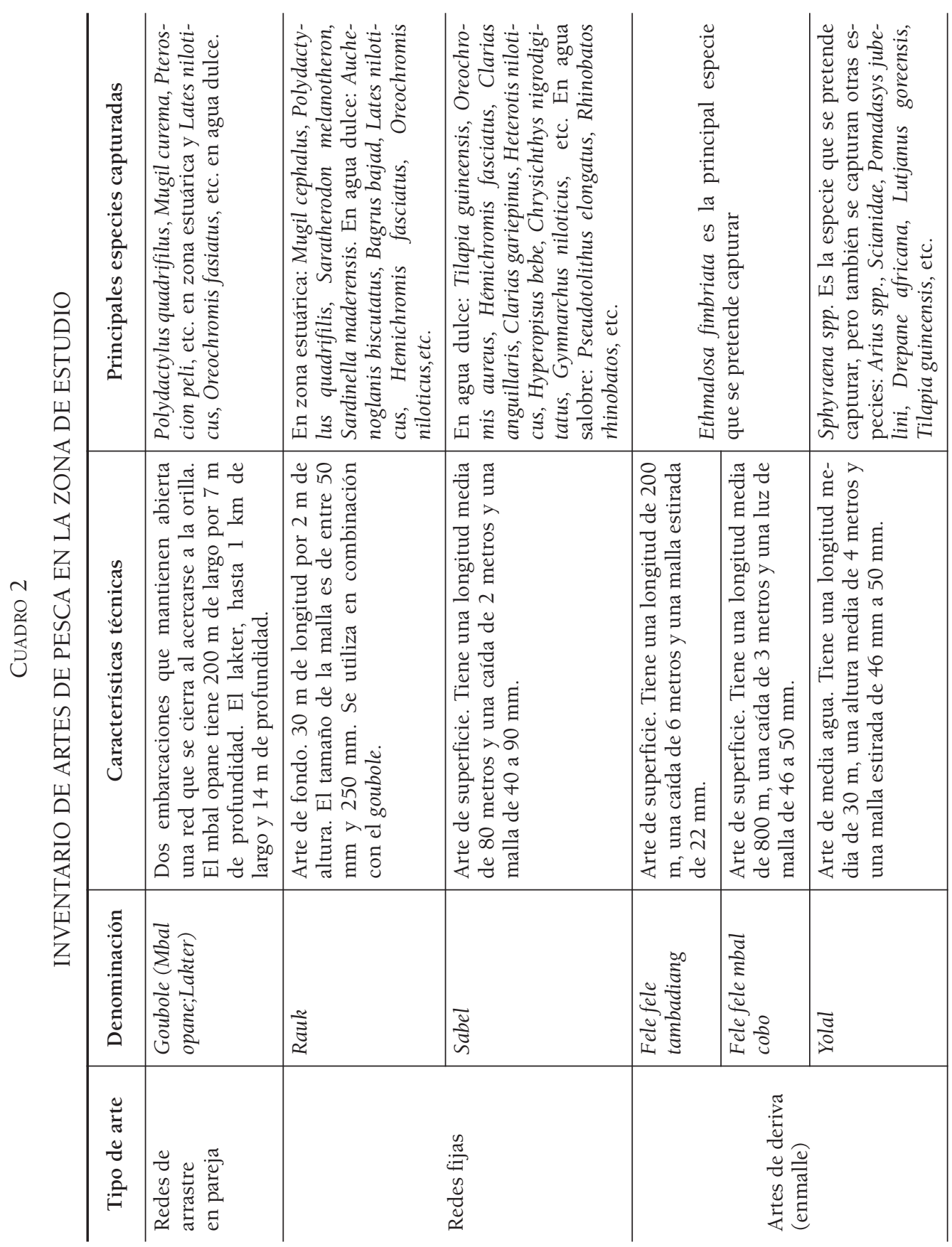

Estudios Geográficos, Vol. LXXVI, 279, pp. 703-731, julio-diciembre 2015 ISSN: 0014-1496, eISSN: 1988-8546, doi: 10.3989/estgeogr.201525 


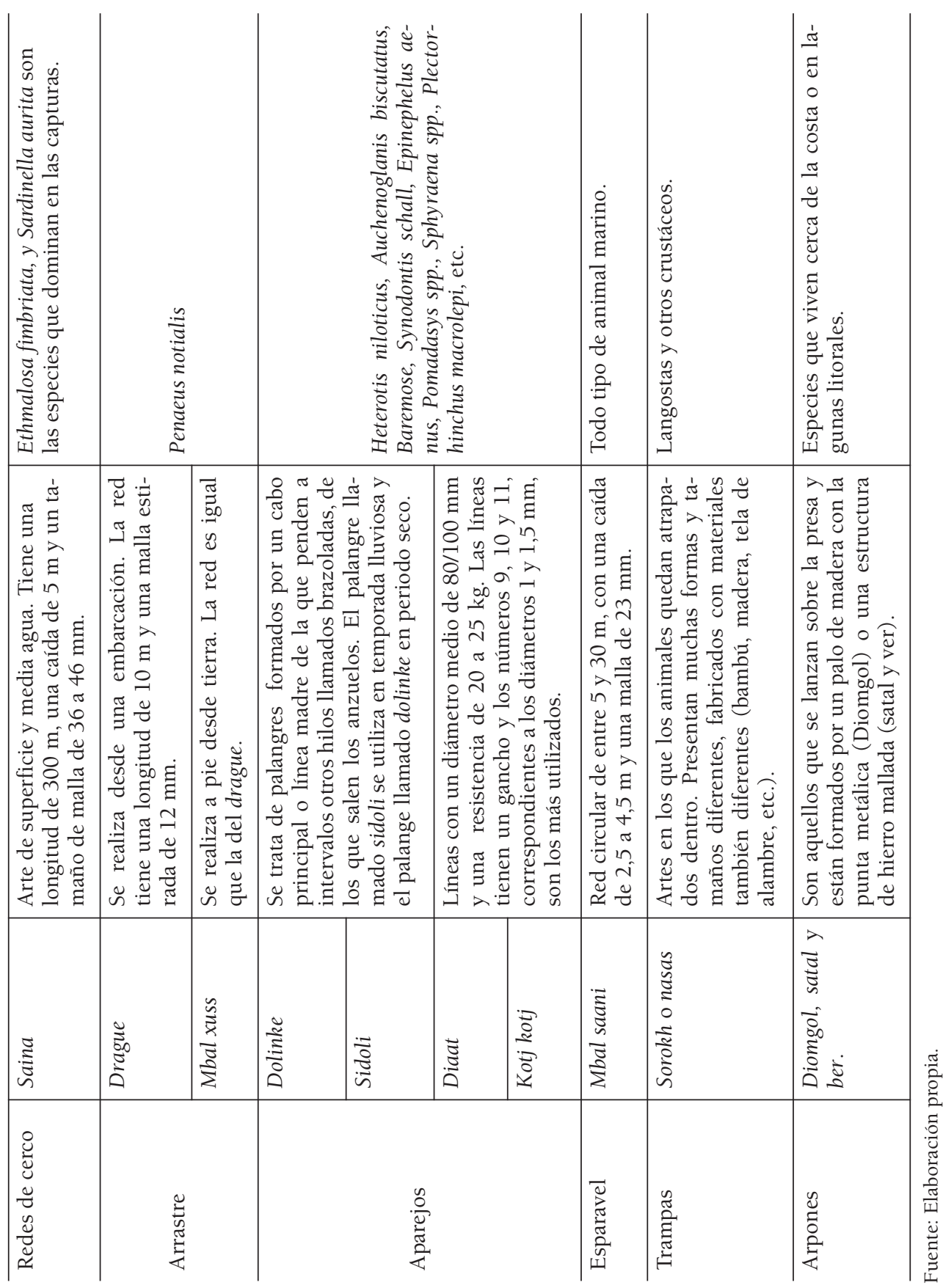

Estudios Geográficos, Vol. LXXVI, 279, pp. 703-731, julio-diciembre 2015 ISSN: 0014-1496, eISSN: 1988-8546, doi: 10.3989/estgeogr.201525 
CUADro 3

EMBARCACIONES DE PESCA EN LA ZONA DE ESTUDIO

\begin{tabular}{lll}
\hline Embarcaciones & \multicolumn{1}{c}{ Características técnicas } & \multicolumn{1}{c}{ Uso en el territorio } \\
\hline Barca de mar & $\begin{array}{l}\text { La barca de mar tiene unos } 15 \mathrm{~m} \text { de } \\
\text { largo, } 2 \mathrm{~m} \text { de anchura y } 1.5 \mathrm{de} \text { pro- } \\
\text { fundidad y una capacidad de carga } \\
\text { media de } 10 \text { tonelaje }\end{array}$ & $\begin{array}{l}\text { La embarcación se utiliza en zona } \\
\text { marítima y estuárica. }\end{array}$ \\
Tara & $\begin{array}{l}\text { La embarcación tiene unos } 9 \mathrm{~m} \text { de } \\
\text { largo, } 1.5 \mathrm{~m} \text { anchura y } 1 \mathrm{~m} \text { de pro- } \\
\text { fundidad. }\end{array}$ & $\begin{array}{l}\text { Se usa exclusivamente en la parte } \\
\text { estuárica. }\end{array}$ \\
Mbul & $\begin{array}{l}\text { El Mbul tiene } 5 \text { a } 9 \mathrm{~m} \text { de largo, 0,5 } \\
\mathrm{m} \text { de anchura y 0,7 } \mathrm{m} \text { de profundi- } \\
\text { dad }\end{array}$ & $\begin{array}{l}\text { Se usa en la parte de agua dulce, } \\
\text { en elte de la presa de Diama. }\end{array}$ \\
\hline
\end{tabular}

Fuente: Elaboración propia.

Con esta variedad de procedimientos de captura, de aspectos ambientales y socioeconómicos muy heterogéneos hemos intentado determinar en la siguiente parte los tipos de ocupación espacial.

Tipología de los artes, horarios de pesca y presiones sobre el medio

De acuerdo con el material de pesca utilizado y su naturaleza, el número de pescadores a bordo de las embarcaciones y las especies que se pretenden capturar, uno de los factores mencionados puede aparecer para limitar o aumentar una agrupación de los artes de pesca en un lugar determinado. Para elaborar nuestra tipología, seleccionamos como criterio el número de artes de cada categoría, independientemente de si son dos equipos diferentes (por ejemplo entre redes de arrastre, fijas, aparejos, etc.) en la misma unidad pesquera o dos unidades (por ejemplo, palangre o línea; artes de deriva de superficie o de media agua) de las distintas pesquerías. El análisis factorial efectuado a partir del programa SPAD muestra que el factor 1 concentra el $90 \%$ de la varianza total mientras que el factor 2 retiene el $10 \%$ de la inercia. El análisis factorial efectuado según las localidades de pescadores y el tipo de artes utilizados permite diferenciar dos tipos de clase que ejercen presiones en el territorio.

En la clase 1 (formada, entre otras, por las poblaciones de Podor, RichardToll, Tiguet, Bountou Bah, Temeye, Ngnith, Foss, Gae, Doue, Guede chantier, 
Guede village) encontramos artes de pesca tales como goubole, rauk, sabel, dolinke y sidole. Este grupo está formado por pescadores en agua dulce que explotan los ecosistemas de agua dulce del lago de Guiers, del canal de la Taouey, de la reserva de agua de Diama, o de los remansos del Djoudj y la agricultura de regadío (arroz, verdura, etc.), la ganadería o el comercio son muy importantes. Los horarios de pesca son variados y la actividad se practica en cualquier momento del día. Sin embargo, de manera general, en la estación seca, las salidas de las embarcaciones de pesca tienen lugar por la mañana entre las $5 \mathrm{~h}$ y $6 \mathrm{~h}$ y van saliendo hasta el mediodía. Sin embargo, por la tarde se registran salidas esporádicas entre las $17 \mathrm{~h}$ y $21 \mathrm{~h}$ entre los meses de octubre y diciembre. Por contra, los regresos se realizan entre las $7 \mathrm{~h}$ y $9 \mathrm{~h}$ y entre las $19 \mathrm{~h}$ y $23 \mathrm{~h}$. Es decir, pescan una media de dos horas diarias entre enero y septiembre y durante la noche entre octubre y diciembre. Las principales especies de pescado capturadas y comercializadas son la tilapia del Nilo (Oreochromis niloticus), el ndiaguel (Heterotus niloticus) y la perca del Nilo (Lates niloticus).

En la clase 2 (formada, entre otras, por las poblaciones de Saint-Louis, Doune Baba Dieye, Mouit, Diel Mbame), encontramos varios tipos de artes (esparavel, fele fele, saina, sabel, rauk, yolal, líneas, trampa, etc.) que explotan y ejercen presión en la zona del estuario y el litoral. La pesca requiere un material bien adaptado a las condiciones hidrológicas estuarianas (mareas, corrientes, viento, profundidad, etc.) y de la variedad de los recursos: Ethmalosa fimbriata, Mugil cephalus, Saratherodon melanotheron, Synaptura cadenati, Polydactylus quadrifilus, Penaeus notialis, etc. Durante la estación seca (octubre a junio), la mayoría de las salidas de pesca se hacen por la tarde o la noche, particularmente entre las $18 \mathrm{~h}$ y $21 \mathrm{~h}$, mientras que durante la temporada de lluvias (julio a septiembre), las salidas tienen lugar durante el día; las vueltas que se realizan por la mañana alrededor de las $7 \mathrm{~h}$ y $8 \mathrm{~h}$ entre noviembre y mayo. Es decir, están pescando entre 12 y 13 horas diarias. Entre junio y julio, las llegadas se registran por la noche. En este grupo, descartamos dos subgrupos que son el centro Saint-Louis que se caracteriza la importancia de la pesca con el esparavel y el pueblo de Dounebaba Dieye quien debido a su proximidad con el canal de la Lengua de Barbarie se diferencia por la pesca con artes fijos de fondo (rauk).

\section{Cambios físico-químicos y biológicos (S)}

Los estudios físico-químicos realizados hasta el momento (Ceeki, 1992; Kane, 1997; Mietton, 2007; Kane, 2010) han demostrado la degradación de la 
calidad de las aguas superficiales en algunas zonas del área de estudio. Por ejemplo, durante el año hidrológico 1990-1992, cuando la presa sólo estuvo cerrada 146 días, la salinidad del agua disminuyó a 22 g/l (Kane, 2010).

De todo lo indicado, en el estuario, nosotros destacamos durante nuestro trabajo de campo que cuando la presa de Diama queda cerrada la calidad del agua en toda la zona que se extiende desde la presa hasta la desembocadura es salada en algunas partes y existen grandes variaciones, a veces bruscas, con concentraciones de la salinidad que puede superar los $36 \mathrm{~g} / \mathrm{l}$ y un $\mathrm{pH}$ de 5,7 en el pueblo de Mouit. La salinización tiene efecto en la degradación de los ecosistemas. Los cambios físico-químicos están ocasionados principalmente por la eutrofización, debido a la reducción de la velocidad de flujo y a la oxigenación del agua, junto a la proliferación de malas hierbas acuáticas, aunque también a la contaminación química y biológica relacionada con el vertido de plaguicidas y aguas residuales al río.

Desde el punto de vista de las especies, durante el trabajo de campo se contabilizaron 69 especies de peces y 28 familias. De esta forma, la biodiversidad del delta y el valle se caracteriza por la predominancia de Bagridae con ocho especies y Characidae con siete especies. Después, les siguen los Mochockidae Mugilidae con cinco especies y el grupo de Cichlidae y Mormyridae con cuatro especies y Carangidae y Clariidae con tres especies. Finalmente, los grupos menos representados son Distichodontidae, Schilbeidae, Clupidae, Portunidae, Centropomidae, Polynemidae y Haemulidae con dos especies cada uno, y Penaeidae, Malapteruridae, Gymnarchidae, Soleidae, Ludjanidae, Cyprinidae, Polypteridae, Ariidae y Osteoglossidae con una especie cada uno.

Ecológicamente, la distribución espacio-temporal ha cambiado considerablemente. Antes de la construcción de la presa de Diama en 1986, en periodo seco, el delta estaba colonizado por especies estuáricas y eurihalinas marinas mientras que en temporada de lluvia la biodiversidad cambiaba radicalmente en cuando llegaban las aguas dulces y las especies marinas desaparecían y eran sustituidas por las especies de agua dulce y todas las especies de agua dulce presentes en el valle bajo se podían encontrar durante las inundaciones en el delta inferior, excepto unas pocas, como Pollimyrus isidori, Raianas senegalensis y Synodontis filamentosus. Ahora con la construcción de la presa de Diama, el área de distribución de las especies estuarianas y marinas pasa a extenderse desde la desembocadura del río hasta la presa de Diama. En el sector sur de la presa de Diama (estuario), en temporada de lluvias, la biodiversidad es más heterogénea y se encuentran especies con afinidad continental, como Chrysichthys nigrodigitatus, Hemichromis fasciatus, Clarias anguillaris y Bagrus bajad, pero también especies marinas y estuarinas como Sarotherodon melanotheron, Ethmalosa fim- 
briata, entre otras. En la época seca, en cuanto la salinidad empieza a ser elevada, notamos la desaparición de todas las especies de agua dulce y la parte norte de la presa de Diama pasa a ser colonizada por las especies de agua dulce. Durante ésta época, no hay especies dominantes en el área de estudio.

\section{Captura por artes de pesca y percepción de los pescadores (I)}

El estudio de impactos permite aprehender la dinámica de la actividad de pesca en la zona, especialmente el impacto de los artes de pesca sobre el recurso. Desde la construcción de las infraestructuras hidroagrícolas la pesca artesanal se encuentra en una situación de disminución de las capturas (figura 1). Posteriormente, se ha demostrado el efecto de las acciones humanas en los cambios, tanto positivos como negativos, de carácter fisicoquímico y biológico. En el área de estudio, siete tipos de pesca (goubole, sabel, rauk, saina, yolal, fele fele y línea) aseguran más de $95 \%$ de las capturas.

La captura media diaria por pesca de redes fijas de fondo (rauk, sabel) para todas las especies es de 36,6 kg en el lago de Guiers y de $5 \mathrm{~kg}$ por el Goubol en la Taouey. Las especies como Tilapia, Labeo, Hyperopisus, Bagrus, Synodontis, Schilbe, Chrysichthys y Clarias spp representan entre el 80 y $90 \%$ de las capturas. En contraste con las capturas en agua dulce, el Ethmalosa fimbriata domina en las capturas a partir del mes de mayo en el sector estuárico. La pesca se hace con el arte de pesca llamado saina, cuyo rendimiento se compone de $77,7 \%$ de Ethmalosa fimbriata y $17,3 \%$ de tilapia y una docena de otras especies. El saina tiene una rentabilidad media diaria de $25 \mathrm{~kg}$ de todas las especies. Por otra parte, tenemos respectivamente fele fele, redes de arrastre de langostino blanco y líneas cuya rentabilidad media es de 21,8 kg. Según los pescadores encuestados en la zona de Gandiole, las capturas medias diarias han bajado desde el año 1986 de más de $30 \mathrm{~kg}$.

Centrándonos en la zona de Gandiole, destacamos que el canal de la Lengua de Barbarie también ha implicado impactos en la actividad pesquera. Con la apertura de ese canal, los pescadores han tenido que cambiar su amarre habitual de la embarcación, a la orilla más alejada del poblado y utilizan el trayecto a través del canal para ir a pescar al mar, ahorrando tiempo y combustible (hasta 20 litros), ya que antes estaban obligados a pasar por la desembocadura natural del río situada a unos $30 \mathrm{~km}$ más al sur. También hemos constatado la recrudescencia de la práctica de la pesca de marisco a lo largo del pueblo de Degouniaye. Por contra, se observan cambios negativos importantes en el suministro de agua potable que es cada vez más difícil debido a la salinización de las aguas freáticas. 
Según la percepción de los pescadores sobre los impactos de la presa de Diama y del canal de la Lengua de Barbarie, estas obras llevaron a la extinción de algunas especies tales como Synodontis synodontis, Bagrus o Synodontis filamentosus en Saint-Louis y Gandiole y Polypterus bechir y Ethmalosa fimbriata en las aguas de Richard-Toll, dónde también han pasado a ser casi inexistentes la anguila africana, Gymnarchus niloticus y Lates niloticus.

De acuerdo con los pescadores en el lago Guiers, el Lates niloticus es ahora uno de los peces más buscados y el más caro; los pescaderos y los comerciantes afirman también que antes de la construcción de las presas algunas especies como Tilapia zilii, Tilapia dageti o Tilapia guineensis eran abundantes en la cuenca del río, pero ahora se ha reducido drásticamente su presencia. Especies como Saratherodon melanotheron, Callinectes amnicolao Marginatus callinectes son muy abundantes en toda la región, aunque se encuentran principalmente en el estuario, mientras que en el norte de la presa de Diama, en el mercado de Richard-Toll, es el Oreochromis niloticus y algunas tilapias las especies más abundantes.

Los pescadores y las mujeres vendedoras de pescado en el valle bajo en Podor y Matam opinan que la situación en la que se encuentra la pesca es muy preocupante porque las presas han tenido un impacto muy negativo sobre los recursos. La presa de Diama ha causado la escasez de ciertas especies tales como Tilapia zillii, Tilapia guineensis, Hydrocinus forskalii, Lates niloticus, Citharinus Citharus, Raimas senegalensis, Clarias anguillaris, etc. Otras especies como la anguila africana, Gymnarchus niloticus y peces disco ya no se encuentran en las aguas dulces de Podor.

\section{Estrategias adaptativas o de mejora (R)}

Con el colapso de las reservas pesqueras y la disminución de las capturas, las respuestas son múltiples y se pueden medir a través de la actitud de los pescadores y de las respuestas institucionales (Bogardi, 2004). En efecto, el seguimiento del calendario de actividades de los pescadores en los puertos de pesca de Saint-Louis, Gandiole, Richard-Toll y Podor (figura 5) permite ver que aparte de la pesca, la mayoría de los pescadores ejercen otras actividades con el fin de conseguir ingresos adicionales. Según nuestra encuesta, el 30\% de de los pescadores en medio continental practican la agricultura (arroz, caña de azúcar, horticultura, etc.) y la ganadería (ovejas, cabras, gallinas, etc.) mientras que en zona estuárica hasta el $10 \%$ de los pescadores se dedican al transporte de turistas (paseo en la isla de Aves del Parque Nacional de Lengua de Barbarie, el faro del Gandiole, antigua desembocadura del río, etc.). La hor- 
ticultura es importante en el delta y se practica especialmente en las zonas húmedas llamadas niayes o depresiones interdunares. Sin embargo, desde la instalación del canal de la Lengua de Barbarie, esta actividad se encuentra en recesión, debido a la salinización del suelo. Las otras causas de la inactividad de los pescadores se deben al descanso relacionado con falta de rentabilidad ( $10 \%$ en los centros de Saint-Louis y Gandiole y $8 \%$ en Richard-Toll y Podor); $\mathrm{u}$ otros motivos tales como el clima o las condiciones hidrológicas ( $8 \%$ en zona estuárica y $10 \%$ en zona de agua dulce).

Hemos constatado durante nuestro trabajo de campo que los pescadores Guet ndar-Guet ndar y walo walo efectúan varias migraciones a lo largo de la cuenca o del litoral marítimo para capturar el pescado. Sin embargo, el fenómeno de la migración de los pecadores de Guet ndar es antiguo. Van Chi Bonnardel (1985) explicó que la instalación de los Guet ndar en la Lengua de Barbarie se remonta al siglo Xvi. Estos pescadores pescaban únicamente en el río y la conquista del mar para la pesca se ha acelerado debido a la introducción de nuevos artes de pesca y a la motorización de las embarcaciones. En general, las migraciones se intensifican y tienen lugar a partir del mes de abril. Las migraciones a lo largo de la cuenca del río son muy importantes: casi el $36 \%$ de los pescadores en agua dulce en los puertos de pesca de Diama, Richard-Toll, Podor y Matam afirman hacer desplazamientos de 1 o 2 días (llamados marea) hacia la presa de Diama, remansos acercanos del parque de Djoudj. En el lago de Guiers, uno de los lugares más importantes del área de estudio, se registra un número muy importante de pescadores venidos de los países fronterizos de Malí y Mauritania, instalados en los pueblos de Temeye y Bountou Bah. En cambio, los pescadores migrantes en zona estuárica (38\% en los pueblos en el Gandiole y Gae) optan por practicar sus actividades en los centros de pesca situados en el litoral marítimo de Senegal (Mbour, Joal, Casamance, Kayar) y en Mauritania y Banjul.

Además de estos tipos de migración (en la cuenca y el litoral marítimo), la emigración internacional es un fenómeno muy preocupante por tratarse mayoritariamente de una actividad ilegal y de muy alto riesgo. Cruzar el Atlántico hacia la costa española se ha convertido en una alternativa para algunos pescadores $\mathrm{u}$ otros habitantes de la región que buscan un futuro mejor. En esta aventura a Europa, los pescadores suelen ser los capitanes. En los pueblos de Mouit y Degouniaye se registraron entre 2006 y 2012 casi 91 emigraciones clandestinas hacia España (Bâ, 2013). Sin embargo, sobre más de 16.000 emigrantes ilegales que llegaron a las costas españolas, cientos de personas murieron durante el viaje y muchos de los que llegaron no consiguieron mejorar sustancialmente su nivel de vida. 


\section{FIGURA 5}

DIAGRAMA DE ACTIVIDADES DES LOS PESCADORES: (A) EN ZONA ESTUÁRICA EN SAINT-LOUIS Y GANDIOLE; (B) EN AGUA DULCE EN RICHARD-TOLL Y PODOR


Fuente: Elaboración propia.

Estudios Geográficos, Vol. LXXVI, 279, pp. 703-731, julio-diciembre 2015 ISSN: 0014-1496, eISSN: 1988-8546, doi: 10.3989/estgeogr.201525 
Las respuestas institucionales a los problemas de la pesca se enfocan esencialmente en la reglamentación de los usos de las artes de pesca y se aplican en general en todo el territorio de Senegal. El Decreto 76-836 del 26 de julio de 1976 define la talla de la malla de los artes de pesca y el código de 1987 además de reglamentar las mallas también redefine el uso de artes. Pero es el artículo 30 del Código de pesca de 1998 el que cambió la dimensión mínima y la prohibición del uso del monofilamento o multimonofilamento en nylon. Hoy en día se observa un aumento progresivo de las medidas mínimas.

Algunas iniciativas piscícolas se desarrollaron en Richard-Toll, Gaya, Ndiarème, Nianga, Guede, Mboumba y Gamadji, con el fin de satisfacer la demanda de pescado en el área. Los experimentos se basaron principalmente en la tilapia y el bagre. A pesar de las enormes posibilidades del área (disponibilidad de agua, alimentos, etc.), la producción de pescado es muy baja (alrededor de $15.000 \mathrm{~kg}$ anuales) por el hecho de la falta de experiencia y de capacidad inversora de los productores.

\section{DisCUSIÓN Y CONCLUSIONES}

Este estudio es una contribución para la gestión de la pesca artesanal en el delta y el valle bajo del río Senegal. Aunque ya se han realizado distintos estudios en diferentes sectores del sistema pesca en el área de estudio, nuestro trabajo se planteó el ejercicio de poner en marcha el marco conceptual DPSIR para relacionar los aspectos ambientales y socioeconómicos de la pesca. Esta tarea no fue fácil, en primer lugar, debido a la especificidad de las cuencas fluviales que se caracterizan por las interconexiones entre los socioecosistemas y los múltiples usos y usuarios que actúan en la zona y con objetivos a menudo contradictorios. En segundo lugar, a fin de tener datos sobre los componentes del DPSIR y comparar el resultado con estudios en el área (Reizer, 1972; Bousso, 1997; Albaret y Diouf, 1994) en la biodiversidad y ecología de los peces, se hizo trabajo de campo durante un ciclo de peca (en temporada seca entre noviembre y julio y en temporada de lluvia entre julio-agosto y octubre). Teniendo en cuenta la complejidad de los sistemas pesqueros, este estudio no pretende ser exhaustivo, pero analiza la actividad de forma global con el fin de proponer soluciones para una mejor gestión de la pesca.

En el contexto de la pesca artesanal en la cuenca del río Senegal, varios aspectos podrían ser elegidos como fuerzas motrices. Los factores socio-económicos con la generalización de la agricultura y la artificialización de la cuenca del río debido a la instalación de represas, canales, diques, etc. han cambiado 
la dinámica de los ecosistemas. Estos fenómenos asociados a los diferentes precios del pescado en los mercados y a la fuerte demanda han sido determinantes para el sector de la pesca y han causado varias presiones sobre los ecosistemas. Hemos contabilizado dieciséis tipos de artes de pesca. Estos artes de pesca son artesanales y su uso depende del periodo del año y la especie o grupo de especies que se pretenden capturar.

Hoy en día, la situación de la pesca está marcada por una evolución regresiva con una caída de las capturas y un aumento de la dependencia en suministro de pescado. Las observaciones realizadas en el área de estudio muestran una disminución de las especies. Entre 2012 y 2013, hemos identificado un número de 68 especies de peces mientras que Reizer (1974) identificó 114 en su censo. La construcción de la presa de Diama, los planeamientos hidroagrícolas y el canal de la Lengua de Barbarie han aumentado la complejidad de la migración de los peces que, en consecuencia, determinan los desplazamientos de los pescadores que en general son más largos de los que se hacían en décadas anteriores (Bousso y Touré, 2008). Actualmente, la dinámica hidrológica ha cambiado y ha dejado de tener un ritmo natural debido al modo de gestión de las presas, lo que aumenta la vulnerabilidad y la dependencia de los pescadores que cada vez asocian más su actividad a la práctica de agricultura o el transporte de turistas para diversificar y aumentar sus ingresos; incluso algunos han optado por la emigración clandestina hacia Europa.

El modelo DPSIR sugiere varios actores (los servicios de agua, pescadores, constructores de embarcaciones, comerciantes de pescado, transportistas, etc.). Según Magrin y Seck (2009), a pesar del contexto de la descentralización política y de desarrollo local iniciado por las autoridades senegalesas, el sector de la pesca artesanal tiene dificultades para revitalizarse, así como los pescadores para hacer oír sus reivindicaciones.

Este estudio pretende haber contribuido a generar un mayor conocimiento científico para favorecer una gestión más sostenible de la pesca artesanal en el área de estudio. En este sentido, partiendo de la situación actual analizada previamente, consideramos que se hace necesaria una gestión o gobernanza basada en el compromiso entre los distintos usos que actúan en la cuenca hidrográfica (agricultura, pesca, ganadería, transporte, producción de energía, etc.). La gestión basada en el compromiso es un escenario en el cual los actores coinciden que el suministro de un servicio (por ejemplo, el abastecimiento de agua para la agricultura o la regulación de la cuenca) tiene un impacto directo sobre otro servicio y sobre la biodiversidad (por ejemplo, la pesca). Según Bennett et al. (2009), estas relaciones pueden ser: a) de tipo unidireccional (ej. el suministro de un servicio de A afecta el nivel de presta- 
ción de un servicio de B, pero no a la inversa) como el caso de las presas de Diama y Manantali, que fueron construidas con el fin de promover la autosuficiencia alimentaria, la producción de energía y el aumento de los ingresos para la producción agrícola intensiva, pero han causado un colapso de la actividad pesquera (y agrícola familiar); o b) de tipo bidireccional (el suministro de un servicio A afecta el nivel de suministro de un servicio B, que a su vez afecta el suministro de A). Sin embargo, desde el punto de vista de la sostenibilidad territorial, el problema identificado es que nos encontramos delante el tipo unidireccional. Hoy en día frente a la generalización de la agricultura de regadío la consideración de todos los intereses de los diferentes usuarios del agua es vital para el equilibro de los ecosistemas, así como desde el punto de vista socio-económico. Acciones como el control del uso de pesticidas, de las plantas invasores y el desarrollo de estudios sobre los impactos de la gestión de las presas sobre los hábitats y las zonas de reproducción de los peces deberían ganar más relevancia en el área de estudio. Los pescadores tienen una experiencia del medio natural y los artes de pesca, sin embargo, la falta de financiación constituye un problema para renovar y modernizar el material de pesca. En definitiva, hace falta poner en marcha sistemas de diálogo entre la administración, los pescadores y otros usuarios del agua, tales como las plataformas multi-sectoriales / usuarios, como los que la FAO ya desarrolla en el Mediterráneo. Esto permitiría tener en consideración el saber de los que explotan directamente los recursos e identificar formas de respuestas más adecuadas frente a las problemáticas socio-ambientales actuales y futuras. Al mismo tiempo, estos ejercicios de gestión participativa permiten una mayor responsabilización de los productores sobre los recursos y se pueden apoyar con las políticas de las áreas protegidas existentes, analizando su papel en este proceso.

Fecha de recepción: 9 de abril de 2014.

Fecha de aceptación: 6 de marzo de 2015.

\section{BiBLIOGRAFÍA}

Albaret, J. J. y Diouf, P. S. (1994): "Diversité des poissons des lagunes et des estuaires ouest-africains”. Afri. Centr. Zool., 275, pp. 165-177.

Bâ, K. (2013): "Apport de la Télédétection et des SIG dans l'étude de l'évolution de la Langue Barbarie et de l'estuaire du fleuve Sénégal". Tesis doctoral inédita, Dakar, Université Cheikh Anta Diop. 
Bennett, E. M.; Peterson, G. D. y Gordon, L. J. (2009): "Understanding relationships among multiple ecosystem services". Ecology Letters, 12, pp. 1-11.

Bidone, E. D. y Lacerda, L. D. (2004): "The use of DPSIR framework to evaluate sustainability in coastal areas. Case study: Guanabara Bay basin, Río de Janeiro, Brazil". Regional Environmental Change, 4/1, pp. 5-16.

Bogardi, J. J. (2004): "Hazards, Risks, and Vulnerabily in a changing environment: the inexpected onslaught on human security?" Global Environmental Change, 14, pp. 361-365.

Bousso, T. (1997): "The estuary of Senegal River: the impact of environnemental changes and the Diama Dam on resource status and fishery conditions", in K. Remane (ed.): African inland fisheries, aquaculture and the environment. Oxford, Blackwell, pp. 45-65.

Bousso, T.; Touré, E. O. y Thiam, N. (2008): "Biodiversité des eaux douces et son processus d'integration dans le domaine de la pêche et de l'aquaculture", en: Rapport de mission. Dakar, Centre de Recherches Oceanografiques de Dakar-Thiaroye.

Camanho, A. S.; Hora, J.; Gaspar, M. B. y Oliveira, M. M. (2010): Analysis of the artisanal fisheries in the Atlantic Arc based on the DPSIR framework. FEUP/IPIMAR Report of Project PRESPO.

Camara, M. M. B. (2008): "Quelle gestion des pêches artisanales en Afrique de l'Ouest? Etude de la complexité de l'espace halieutique en zone littorale sénégalaise". Tesis doctoral inédita. Dakar, Université Cheikh Anta Diop.

Cecchi, P. (1992): Phytoplancton et condition du milieu dans l'estuaire du fleuve Sénégal: effet du barrage de Diama. Montpellier, Université de Montpellier 2.

Corlay, J. P. (1993): "Les concepts d'espace halieutique: réflexion de géographes sur les pêches maritimes à partir du cas danois", en Colloques et séminaires : Question sur la dynamique de l'exploitation halieutique. Montpellier, ORSTOM/ IFREMER, pp. 125-140.

Diarra, I. (2003): "Aires protégées et gestion des resources naturelles", en: DEA CHAIRE UNESCO de Géographie. Dakar, Université Cheikh Anta Diop.

Elliot, M. (2002): "The role of the approach and conceptual models in marine: an example of offshore wind power". Marine Pollution Bulletin, 44, pp. III-VII.

European Environmental Agency (EEA) (1999): Environmental indicators: Typology and overview. Copenhagen, EEA Technical report no. 25.

Faye, A. (1996): "Les critères de gestion optimum des ressources en eau dans le delta du fleuve Senegal". Tesis doctoral inédita. Dakar, Université Cheikh Anta Diop.

Gordon, H. S. (1954): "The economic theory of a common property resource". The fishery Journal of Political Economy, 62/2, pp. 124-142.

Hardin, G. (1968): "The tradedy of the commons". Science, 162, pp. 1243-1247.

Kane, A. (1957): "Laprès barrage dans la vallée du fleuve Sénégal Modification hydrologique, morphologiques, géochimiques et sédimentologiques. Conséquences sur le milieu naturel et les aménagements hydro-agricoles". Tesis doctoral inédita, Dakar, Université Cheikh Anta Diop. 
Kane, C. (2010): "Variabilité du système socio-environnement du domaine Sahélien: l'exemple de l'estuaire du fleuve Sénégal. De la perception à la gestion des risques", Tesis doctoral inédita. Strasbourg, Université de Strasbourg.

Le Fur J.; Laloe F; Durand, M. H. y Chaboud C. (1999): "Co-viabilité des systèmes halieutiques”. Nature, Sciences, Sociétés, 7/2, pp. 19-32.

Lin, T., Xue, X. y Lu, C. (2007): "Analysis of Coastal Wetland Changes Using the 'DPSIR' Model: A Case Study in Xiamen, China”. Coastal Management, 35, pp. 289-303.

Magrin, G. y Seck, S. M. (2009): "La pêche continentale en sursis? Observation sur les pêcheries en rive gauche de la vallée du Sénégal dans un contexte de décentralisation". Geocarrefour, 84/1-2, pp. 54-64.

Mangi, S. C., Robert, C. M. y Rodewell, L. D. (2007): "Reef fisheries management in Kenya: Preliminary approach using the driver - pressure - state - impacts - response (DPSIR) scheme of indicators". Ocean E Coastal Management, 50, pp. 463-480.

Maxim, L.; Spangenberg, J. H. y O'Connor, M. (2009): "An analysis of risks for biodiversity under the DPSIR framework". Ecological Economics, 69, pp. 12-23.

Mbaye, A. (2002): "Analyse sociologique de la differenciation technique dans la pêche artisanale maritime sénégalaise", en: Séminaire C3ED-OA, LINUS, Dakar.

Mietton, M.; Dumas, D.; Hamerlunck, O. et al. (2007): "Le delta du fleuve Sénégal. Une gestion de l'eau dans l'incertitude chronique", en Paul Allard, Dennis Fox, Bernard Picon (eds.): Incertitude et Environnement. La fin des certitudes scientifiques. Aix en Provence, Editions Edisud, pp. 321-336.

Monteillet, J. (1988): "Environnements sédimentaires et paléoécologie du delta du Sénégal au Quaternaire". Tesis doctoral inédita, Perpignan, Université de Perpignan.

OMVS (2005): Etude d impact de louverture d une embouchure artificielle à laval de Saint-Louis sur le barrage de Diama. Dakar, Rapp final.

Organization for Economic Cooperation and Development (1994): Environmental indicators: OECD core set. Paris, OECD.

Quensiere, J. (1993): "De la mondialisation à la gestion systèmique des pêches". Nature, Sciences, Société, 3, pp. 211-220.

Reizer, C. (1974): "Définition d'une politique d'aménagement des ressources halieutiques d'un écosystème aquatique complexe par l'étude de son environnement abiotique et anthropique. Le Sénégal moyen et inférieur". Tesis doctoral inédita. Arlon, Fondation Universitaire Luxembourgeoise.

Reizer, C. y Lessent, P. (1972): "Les pêches continentales du bas Sénégal". Revue Bois et Foret des Tropiques, 143, pp. 3-21.

Rigler, F. H. (1982): "The relation between fisheries management and limnology". Transactions of the American Fisheries Society, 11/2, pp. 121-132.

Sall, M. (1984): "Dynamique et morphogenèse actualle au Sénégal occidental". Tesis doctoral inédita, Strasbourg, Université Louis Pasteur.

Schaefer, M. B. (1957): "Some considerations of population dynamics and economics in relation to the management of the commercial marine fisheries". Journal of the fisheries Research Board of Canada, 14/5, pp. 669-681. 
Service Regional de la Statistique et de la Demographie (SRSD) (2009): Situation economique et sociale de la región de Saint-Louis, edition 2009. Saint-Louis.

Svarstad, H.; Petersen, L. K.; Rothman, D.; Siepel, H. y Waitzold, F. (2008): "Discursive biases of the environmental research framework DPSIR". Land use Policy, 25/1, pp. 116-125.

Touré, E. O.; Diop, E. O. y Kane, A. (2008): "La fleche littorale de la Langue de Barbarie: un espace de risque conflictuel pour les activités de pêche et tourisme", in: Actas del Coloquio Internacional pluridisciplinar "Le littoral: Subir, Dire, Agir". Lille, Universidad de Lille 1.

Touré, E. O. y Breton, F. (2013): “Cooperación transfronteriza en la esfera del agua: el caso del rio Senegal”, en Marti Boada y Roser Maneja (dir.): El agua y la vida: cooperación en la esfera del agua. Barcelona, CSIC/Lunwerg Editores, pp. 202-209.

Touré, E. O. y Romagosa, F. (2013): "El impacto del turismo en la Lengua de Barbarie (Delta del río Senegal)". Cuadernos de Turismo, 31, pp. 289-309.

Union Internationale pour la Conservation de la Nature (UICN) (2006): Evaluation économique des ressources sauvages au Sénégal. Dakar, UICN.

Van Chi Bonnardel, R. N. (1985): Vitalité de la petite pêche tropicale, pêcheurs de SaintLouis du Sénégal. Paris, Éditions CNRS.

\title{
RESUMEN
}

Durante más de dos décadas, el delta y el valle bajo del río Senegal han sufrido una grave sequía. Las condiciones climáticas han tenido graves consecuencias en el funcionamiento hidrológico. Además, la artificialización de la cuenca debido a la construcción de infraestructuras hidroagrícolas ha causado otros impactos. Las observaciones realizadas durante un ciclo anual de pesca nos permiten implementar el marco conceptual «Driving forces, Pressures, State, Impacts, Responses" (DPSIR). El artículo pretende contribuir a generar indicadores naturales y socioeconómicos aplicables a la pesca artesanal, así como realizar propuestas para una gestión integrada de la actividad pesquera en el área de estudio.

PAlabras Clave: Delta y valle bajo del río Senegal; pesca artesanal; artes de pesca; indicadores; marco conceptual DPSIR.

\begin{abstract}
For over two decades, the delta and the lower valley of the Senegal River have suffered a severe drought. Weather conditions have had serious consequences on the hydrological functioning. In addition, the artificiality of the basin due to the construction of hydro-agricultural infrastructures has caused other impacts. Observations made during an annual fishing cycle allowed us to implement the conceptual framework "Driving forces, Pressures, State, Impacts, Responses" (DPSIR). This article intends to help generate natural and socioeconomic indicators applicable to artisanal fisheries and make proposals for an integrated fisheries management in the study area.
\end{abstract}


KEY WORDS: Delta and lower valley of Senegal River; artisanal fishing; fishing techniques; indicators; conceptual framework DPSIR.

\section{RÉSUMÉ}

Durant plus de deux décennies, le delta et la basse vallée du fleuve Sénégal est affecté par une grave sécheresse. Les conditions climatiques ont eu de graves conséquences sur le fonctionnement hydrologique. Lartificialisation du bassin consécutive à la construction d'infrastructures hydroagricoles ont engendré d'autres impacts. Les observations réalisées sur le terrain durant un cycle annuel de pêche ont permis d'analyser l'activité selon le cadre conceptuel "Driving forces, Pressures, State, Impacts, Responses" (DPSIR). L'article a pour but de contribuer à générer des indicateurs naturels et socioeconomiques applicables à la pêche artisanale et de faire des propositions pour une gestion intégrée de l'activité de pêche dans la zone d'étude.

Mots CLÉs: Delta et basse vallée du fleuve Sénégal; pêche artisanale; engins de pêche; indicateurs; cadre conceptuel DPSIR. 